Notre Dame Journal of Formal Logic

Volume 29, Number 4, Fall 1988

\title{
There are Denumerably Many Ternary Intuitionistic Sheffer Functions
}

\author{
DJORDJE ČUBRIĆ*
}

In [1] Došen asks what is the number of mutually nonequivalent ternary indigenous Sheffer functions for $\{\rightarrow, \wedge, \vee, \neg\}$ in the intuitionistic propositional calculus (IPC). The answer is: denumerably many.

Following [2] we shall say that a set of functions $F$ is an indigenous Sheffer set for a set of functions $G$ iff every member of $G$ can be defined by a finite number of compositions from the members of $F$ and vice versa. A function $f$ is an indigenous Sheffer function for $G$ iff $\{f\}$ is an indigenous Sheffer set for $G$. The $n$-ary propositional functions $f_{1}$ and $f_{2}$ are mutually equivalent iff for some permutation $P$ of the sequence $A_{1}, \ldots, A_{n}$ in the propositional calculus we can prove $f_{1}\left(A_{1}, \ldots, A_{n}\right) \leftrightarrow f_{2}(P)$. We work all the time in IPC. Expressions of the form $\vdash A$ (or $H A$ ) mean that $A$ is provable (or unprovable) in IPC.

Kuznetsov [3] and Hendry [2] have shown that there is no binary indigenous Sheffer function for $\{\rightarrow, \wedge, \vee, \neg\}$ in IPC. The first example of a ternary indigenous Sheffer function was given in [3]. Here we use one of the three examples given in [1].

The Rieger-Nishimura Lattice of one variable $X, \operatorname{RNL}(X)$ is recursively defined as follows: $P_{0}(X)=X \wedge \neg X, P_{1}(X)=X, P_{2}(X)=\neg X, P_{\infty}(X)=$ $X \rightarrow X, P_{2 n+3}(X)=P_{2 n+1}(X) \vee P_{2 n+2}(X), P_{2 n+4}(X)=P_{2 n+3}(X) \rightarrow P_{2 n+1}(X)$, for $n \geq 0$. For every $i>j, H P_{i}(X) \rightarrow P_{j}(X)$ (see [5] or [4]).

First, we have one simple lemma:

\section{Lemma For every $i \geq 5$ :}

(1) $\vdash \neg X \wedge P_{i}(X) \leftrightarrow \neg X$

(2) $\vdash P_{i}(\perp)$.

Proof: (1) For every $i \geq 5$, we have $\neg X \vdash P_{i}(X)$ directly from $\operatorname{RNL}(X)$. We obtain (2) by using $\vdash \neg \perp$ and (1).

\footnotetext{
*I wish to thank Kosta Došen for the help he gave me in writing this note.
} 
Next, we give the following definition:

Definition $f_{i}(A, B, C)=t(A, B, C) \wedge P_{i}(B \wedge C)$, where $t(A, B, C)=((A \vee$ $B) \leftrightarrow(C \leftrightarrow \neg B)) \vee(A \leftrightarrow(C \leftrightarrow \neg B))$ (see [1]), and $P_{i}(B \wedge C) \in \operatorname{RNL}(B \wedge C)$.

Theorem For every $i \geq 5, f_{i}$ is an indigenous Sheffer function for $\{\rightarrow, \wedge$, $\vee, \neg\}$ and for every $i \geq 5$ and every $j>i, \forall f_{i} \leftrightarrow f_{j}$.

Proof: For every $i \geq 5, f_{i}$ is an indigenous Sheffer function for $\{\rightarrow, \wedge, \vee, \neg\}$ because:

$$
\begin{aligned}
& f_{i}(A, A, A) \leftrightarrow t(A, A, A) \wedge P_{i}(A) \\
& \leftrightarrow \neg A \wedge P_{i}(A) \\
&\leftrightarrow \neg A \quad \text { (by using Lemma }(1)) ; \\
& f_{i}(A, B, \neg B) \leftrightarrow t(A, B, \neg B) \wedge P_{i}(B \wedge \neg B) \\
& \leftrightarrow(A \vee B) \wedge P_{i}(\perp) \\
& \leftrightarrow A \vee B \quad(\text { by using Lemma (2)); } \\
& f_{i}(A, \neg(A \vee \neg A), B) \leftrightarrow t(A, \perp, B) \wedge P_{i}(\perp \wedge B) \\
& \leftrightarrow(A \leftrightarrow B) \wedge P_{i}(\perp) \\
&\leftrightarrow(A \leftrightarrow B) \quad \text { (by using Lemma }(2)) ;
\end{aligned}
$$

and we know that $\{\leftrightarrow, \vee, \neg\}$ is an indigenous Sheffer set for $\{\rightarrow, \wedge, \vee, \neg\}$ (we have: $\vdash(A \rightarrow B) \leftrightarrow((A \vee B) \leftrightarrow B), \vdash(A \wedge B) \leftrightarrow((A \vee B) \leftrightarrow(A \leftrightarrow B)))$.

If for some $i \geq 5$ and some $j>i$, $\vdash f_{i} \leftrightarrow f_{J}$, we have $\vdash f_{l}(\perp, B, B) \leftrightarrow$ $f_{j}(\perp, B, B)$ which implies $\vdash T \wedge P_{l}(B) \leftrightarrow T \wedge P_{j}(B)$, and that implies $\vdash P_{i}(B) \leftrightarrow$ $P_{J}(B)$, which is a contradiction.

Note that this theorem is also valid for $i=3$.

For every $i \geq 5$ and $j>i, f_{i}$ and $f_{j}$ are mutually nonequivalent because $f_{i}(A, B, C)$ is classically equivalent only with $f_{j}(C, B, A)$, but not in IPC (if it is, we have: $\vdash f_{i}(\perp, T, C) \leftrightarrow f_{j}(C, T, \perp)$; then $\vdash t(\perp, T, C) \wedge P_{i}(C) \leftrightarrow T$, and then $\vdash P_{i}(C)$, which is a contradiction). Since we have at most denumerably many nonequivalent ternary indigenous Sheffer functions (consider them as words in the alphabet $\{A, B, C, \rightarrow, \wedge, \vee, \neg\})$, we may conclude that there are exactly denumerably many of them.

For every $n>3$, there exist denumerably many $n$-ary Sheffer functions for $\{\rightarrow, \wedge, \vee, \neg\}$ (we substitute $A_{1} \wedge \ldots \wedge A_{n-2}$ for $A$ in $f_{i}$ ).

We conclude this note with two questions:

(1) Is it true that for every ternary Sheffer function in the classical propositional calculus there exists a classically equivalent function which is a Sheffer function in IPC?

(2) What structure is produced by all ternary Sheffer functions in IPC?

\section{REFERENCES}

[1] Došen, K., "An intuitionistic Sheffer function," Notre Dame Journal of Formal Logic, vol. 26 (1985), pp. 479-482.

[2] Hendry, H. E., "Does IPC have a binary indigenous Sheffer function?," Notre Dame Journal of Formal Logic, vol. 22 (1981), pp. 183-186. 
[3] Kuznetsov, A. V., “Analogi 'shtrikha Sheffera' v konstruktivnoi logike,” Doklady Akademii Nauk SSSR, vol. 160 (1965), pp. 274-277.

[4] Nishimura, I., "On formulas of one variable in intuitionistic propositional calculus," The Journal of Symbolic Logic, vol. 25 (1960), pp. 327-331.

[5] Rieger, L., "On the lattice theory of Brouwerian propositional logic," Acta Facultatis Rerum Naturalium Universitatis Carolinae, vol. 189 (1949), pp. 1-40.

Matematički Institut

Knez Mihailova 35

Beograd, Yugoslavia 
